\title{
An on-line solid phase extraction procedure for the routine quantification of caspofungin by liquid chromatography-tandem mass spectrometry
}

\author{
Fabian Kirchhoff',*, Barbara Maier ${ }^{1}$, \\ Christina Rieger ${ }^{2}$, Helmut Ostermann ${ }^{2}$, \\ Ute Spöhrer $^{3}$ and Michael Vogeser ${ }^{1}$ \\ ${ }^{1}$ Institute of Clinical Chemistry, Hospital of the University \\ of Munich, Munich, Germany \\ ${ }^{2}$ Department of Internal Medicine III, Hospital of the \\ University of Munich, Munich, Germany \\ ${ }^{3}$ Hospital Pharmacy, Hospital of the University of Munich, \\ Munich, Germany
}

\begin{abstract}
Background: Extensive sets of data are required to investigate the potential use of a therapeutic drug monitoring with individualization of dosage of the antimycotic compound caspofungin. The goal was to develop an improved liquid chromatography-tandem mass spectrometry (LC-MS/MS) method for this aim.

Methods: Following protein precipitation, on-line solid phase extraction was performed for sample preparation. As the internal standard compound the veterinary drug tylosin was used. A standard validation protocol was applied.

Results: Good reproducibility and accuracy of the method were observed. On-line solid phase extraction resulted in a convenient work-flow and good robustness of the method.

Conclusions: This improved LC-MS/MS method was found reliable and convenient. It can be suggested for further work on the clinical pharmacology of caspofungin in the setting of clinical research laboratories.
\end{abstract}

Keywords: caspofungin; liquid chromatography-tandem mass spectrometry (LC-MS/MS); on-line solid phase extraction (SPE); tylosin.

Invasive fungal infections represent a leading cause of morbidity and mortality in immunocompromized patients today.

\footnotetext{
*Corresponding author: Fabian Kirchhoff, Hospital of the University of Munich, Institute of Clinical Chemistry, Marchioninistraße 15, D-81377 Munich, Germany Phone: +49 897095 3248, Fax: +49 8970956220 , E-mail: fabian.kirchhoff@med.uni-muenchen.de Received July 12, 2011; accepted November 3, 2011; previously published online November 30, 2011
}

During the last decade the therapeutic armamentarium has expanded substantially and now includes almost 10 compounds to be considered for prophylaxis and treatment of invasive fungal infections (1). Therapeutic drug monitoring (TDM) is increasingly discussed for each of these drugs, however - in contrast to immunosuppressants used in transplantation medicine - guidelines for TDM of anti-mycotic drugs have scarcely been defined or evaluated (2).

For the triazole compounds (itraconazol, posaconazol, voriconazol) chromatographic and mass spectrometric methods are now available in a substantial number of clinical research centers; the analytics of the more novel group of echinocandines has been addressed only by a few groups so far. Among the echinocandines, on the one hand caspofungin plays the most important role in clinical practice to date, on the other hand a higher incidence of side-effects and potential for drugdrug interactions is known for caspofungin (3).

Caspofungin steady-state concentrations in plasma between 0.25 and $2.5 \mathrm{mg} / \mathrm{L}$ have been described for different administration regimens (4); clinically relevant caspofungin levels range up to approximately $10 \mathrm{mg} / \mathrm{L} \mathrm{(5).}$

The aim of our work was to develop a reliable liquid chromatography-tandem mass spectrometry (LC-MS/MS) method for respective clinical investigations, applicable in the setting of routine laboratories, thereby addressing some limitations of previously published methods (see online data Supplemental Appendix 1). Beyond published work on caspofungin measurement by LC-MS/MS two issues were addressed by our work in particular: investigation of a suited and commercially available compound for internal standardization and development of a semi-automated sample preparation protocol based on on-line solid phase extraction (SPE) prior to a standard LC-MS/MS system. This principle has been used in our laboratory for over 10 years now and is found to enable very robust methods with short run times and minimal down-times required for maintenance.

Twenty-five microliters of calibrator, quality control sample, or serum was shortly vortexed with $50 \mu \mathrm{L}$ of phosphate buffered saline (PBS) solution in $1.5 \mathrm{~mL}$ polyproylene cups. Thereafter $75 \mu \mathrm{L}$ of internal standard (IS) working solution [approx. $100 \mu \mathrm{g} / \mathrm{L}$ tylosin in acetonitrile/methanol (9:1 by volume)] was added. Cups were shaken for 10 min using a horizontal shaker. This was followed by centrifugation for $10 \mathrm{~min}$ at $15,000 \mathrm{~g}$. Ten microliters of each supernatant was injected into the LC-MS/MS system (Waters Alliance 2795 
HPLC module; Waters, Milford, MA, USA), coupled splitless to a Micromass Quattro Micro tandem mass spectrometry system; mass spectrometric conditions are listed in the online data Supplemental Appendix 4. Sample clean-up was performed online using on-line SPE.

Supernatant was injected and loaded onto a Waters Oasis HLB column $(2.1 \times 20 \mu \mathrm{mm} ; 25 \mu \mathrm{m}$ particle size; Waters, Milford, MA, USA) in valve position A (scheme of the online SPE is given in Figure 1). The mobile phase was water/ methanol 90/10 (v/v), delivered at a flow rate of $4 \mathrm{~mL} / \mathrm{min}$. Potential interfering compounds were washed into waste. In parallel, the analytical column (xTerra C-8, 2.1 $\times 50 \mathrm{~mm}$; $3.5 \mu \mathrm{m}$ particle size; Waters, Milford, MA, USA) was equilibrated with 70/30 (v/v) acetonitrile/15 mM ammonium formate, delivered at a flow rate of $0.2 \mathrm{~mL} / \mathrm{min}$. After $1 \mathrm{~min}$, the switching valve was changed to position $\mathrm{B}$; the extraction column was now eluted in a back-flush mode onto the analytical column. After another $2 \mathrm{~min}$, the valve was switched back to position A. During the analytical chromatography into the mass spectrometer in position $\mathrm{A}$, the extraction column was washed for 1 min with 80/20 (v/v) methanol/1\% formic acid at a flow rate of $4 \mathrm{~mL} / \mathrm{min}$. The total run time was $3.5 \mathrm{~min}$.

For quantification, we applied $1 / \mathrm{x}$ fit weighting of the peak area ratios of analyte/IS vs. the concentration to obtain the calibration curves.

Calibrators were made by diluting the working solution of caspofungin (see online data Supplemental Appendix 5) with drug-free human serum to obtain caspofungin concentrations of 20.0, 10.0, 5.0, 2.5, 1.25, 0.62 and $0.31 \mathrm{mg} / \mathrm{L}$. In the same manner, we prepared quality control samples in three concentration levels (16.0, 1.6 and $0.4 \mathrm{mg} / \mathrm{L})$. We used one serum pool, in order to additionally study the imprecision of the method. This pool was prepared from residual serum sent for standard clinical chemistry analyses from patients, treated with caspofungin. Aliquots of quality control samples, calibrators and serum pool were stored in polypropylene vials at $-80^{\circ} \mathrm{C}$.

In 11 analytical series, applying full calibration, we demonstrated mean accuracy ranged from $94.0 \%$ to $97.3 \%$ and coefficients of variation (CV) below $8 \%$ (complete data is shown in online data Supplemental Appendix 2). The coefficient of determination of the seven calibrator samples was $>0.99\left(\mathrm{R}^{2}\right)$ in all series (average y-intercept and slope values: $\mathrm{y}=1.01 \mathrm{x}-0.03157)$.

Lower- and upper limit of quantification (LLOQ, ULOQ) were assessed by analyzing different concentrations of spiked drug-free serum in duplicate in three runs. LLOQ was $0.25 \mathrm{mg} / \mathrm{L}$ and ULOQ was $40 \mathrm{mg} / \mathrm{L}$ for caspofungin (criterion for acceptance: accuracy of $80 \%-120 \%$, precision with a $\mathrm{CV}<20 \%$ ).

Specificity of the method was demonstrated, when analyzing 50 samples from residual serum of intensive care patients. None of the samples showed peaks in the multiple reaction monitoring (MRM) trace of caspofungin.

Ion-suppression characteristics of the method were assessed by connecting a T-piece between the chromatographic column and the ion source. Using a syringe pump, a pure solution of caspofungin (approx. $1 \mathrm{mg} / \mathrm{L}$ ) and tylosin $(0.1 \mathrm{mg} / \mathrm{L}$ ) was delivered with a constant flow rate of $10 \mu \mathrm{L} / \mathrm{min}$ into the T-piece. By this post-column infusion, a baseline offset was generated in the MRM-traces of caspofungin and tylo$\sin$. At the respective retention times we did not observe any significant variation of this offset upon injection of human serum samples, ruling out relevant ion suppression by residual matrix components.

To verify recovery of the method, aliquots of a serum pool, from patients treated with caspofungin, was spiked with different amounts of caspofungin working solution, resulting in five different concentration ranges. Quantification in the absence and presence of addition of caspofungin working
A

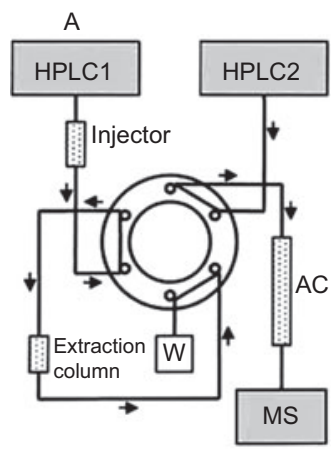

B

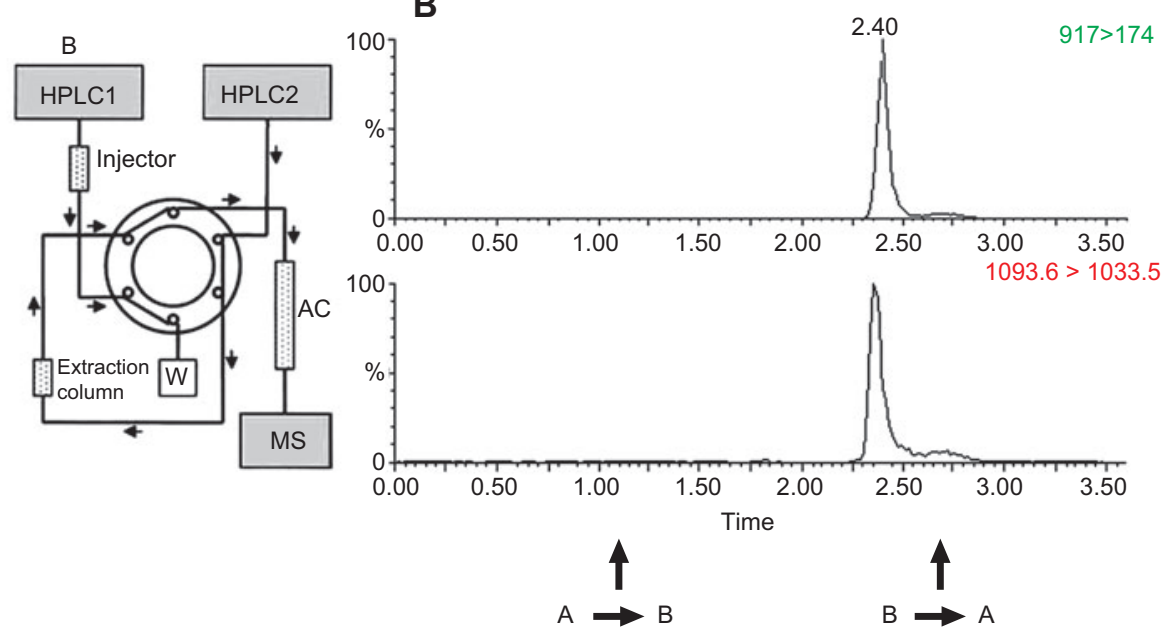

Figure 1 (A) Scheme of the on-line solid phase extraction system (AC, analytical column; W, waste; MS, mass spectrometry system). (B) Representative MRM chromatograms obtained from a pooled serum sample (caspofungin approx. $2.5 \mathrm{mg} / \mathrm{L}$ ). Upper panel: tylosin. Lower panel: caspofungin (retention time approx. $2.35 \mathrm{~min}$ ); total run time: $3.5 \mathrm{~min}$. Arrows indicate switching valve positions. 
solution resulted in a mean recovery rate of $92.5 \%$ (range: $83.3 \%-99.3 \%$ ).

Recovery of the two-step extraction process was evaluated. Samples in three concentration levels were analyzed with and without the on-line SPE procedure. A pure solution of caspofungin in $20 \%$ human serum albumin was directly injected onto the analytical column, by omitting the on-line SPE procedure, whereas spiked serum samples in the same concentration levels were submitted to the entire analytical process. A mean signal recovery of $111.7 \%$ was found $(n=9)$.

Carry-over was evaluated by injecting the highest QC sample 10 times, immediately followed by a blank sample. The chromatogram of this sample was inspected for peaks in the MRM trace of caspofungin and resulted in a carry-over effect of $<1 \%$.

Stability of precipitation extracts was evaluated by reanalysis of deproteinized samples of a calibration series, $24 \mathrm{~h}$ after their initial analysis. Extracts obtained by protein precipitation were found stable for at least $24 \mathrm{~h}-$ a mean deviation of $105.5 \%$ of the peak areas of the MRM trace of caspofungin was noted $(n=6)$.

In this article a convenient LC-MS/MS method for quantification of caspofungin in serum is described; validation data characterize the method as applicable for clinical research use. Regarding previously described LC-MS/MS methods for the quantification of caspofungin in blood, our method is novel with respect to the IS compound and with respect to a convenient semi-automated sample preparation protocol: in three of the previous methods (6-8) an isoster of caspofungin is used as the IS. This compound may be suited, but was obtained from the manufacturer of caspofungin and is not commercially available; thus the method cannot easily be reproduced. Decosterd et al. (9) rely on stable isotope labeled voriconazole for internal standardization. This is questionable as well, since molecular weight and molecular structure of this compound is completely different from that of caspofungin. The compound tylosin, however, has a heterocyclic structure, as is the case for caspofungin, and a similar molecular weight (see online data Supplemental Appendix 3). It is commercially available and not in use in human medicine, but exclusively in veterinary medicine. Therefore, we considered tylosin to be a good candidate for use as IS; this was confirmed by our validation results. Egle et al. (10) completely abdicate the use of an IS, which is very uncommon in clinical mass spectrometry. Also, a complex and fully automated LC-ion trap MS method for caspofungin quantification in serum, which requires two switching valves, two gradient HPLC pumps, a complicated chromatographic set-up and very extended chromatographic run times is described. In our experience, such application of crude serum to on-line extraction cartridges may be convenient but is hampered by the very limited life time of the extraction materials. Applying protein precipitation prior to simple on-line SPE, an extended performance of the extraction columns and - compared to direct analysis of deproteinized samples to HPLC - very good ionization efficacy is guaranteed.

In agreement with previous articles, we identified three particular problems for quantification of caspofungin using
LC-MS/MS: linearity of response over a wider calibration range $(6-8,10)$; adsorption of the analyte to surfaces $(7,11-13)$; and asymetrical peak shape due to isomers of the analyte $(8,10)$. Regarding linearity issues we found dilution with PBS necessary to obtain acceptable calibration functions. Adsorption problems as carry-over issues should be addressed by rather long washing cycles and high proportions of organic solvents. Sub-optimum peak shape due to isomers (as demonstrated in Figure 1) requires consistent peak integration criteria.

In summary we suggest the use of tylosin as an IS together with on-line SPE after protein precipitation as an alternative for quantification of caspofungin in blood using LC-MS/MS.

\section{Conflict of interest statement}

Authors' conflict of interest disclosure: The authors stated that there are no conflicts of interest regarding the publication of this article.

Research funding: None declared.

Employment or leadership: None declared.

Honorarium: None declared.

\section{References}

1. Smith J, Andes D. Therapeutic drug monitoring of antifungals: pharmacokinetic and pharmacodynamic considerations. Ther Drug Monit 2008;30:167-72.

2. Andes D, Pascual A, Marchetti O. Antifungal therapeutic drug monitoring: established and emerging indications. Antimicrob Agents Chemother 2009;531:24-34.

3. Eschenauer G, Depestel DD, Carver PL. Comparison of echinocandin antifungals. Ther Clin Risk Manag 2007;31:71-97.

4. Stone JA, Holland SD, Wickersham PJ, Sterrett A, Schwartz M, Bonfiglio C, et al. Single- and multiple-dose pharmacokinetics of caspofungin in healthy men. Antimicrob Agents Chemother 2002;46:739-45.

5. Keating GM, Jarvis B. Caspofungin. Drugs 2001;61:1121-9.

6. Chavez-Eng CM, Schwartz M, Constanzer ML, Matuszewski BK. Determination of a cyclic hexapeptide, a novel antifungal agent, in human plasma by high-performance liquid chromatography with ion spray and turbo ion spray tandem mass spectrometric detection. J Chromatogr B Biomed Sci Appl 1999;721:229-38.

7. Rochat B, Bolay S, Pascual A, Calandra T, Marchetti O. Liquid chromatography-mass spectrometry method for quantification of caspofungin in clinical plasma samples. J Mass Spectrom 2007;42:440-9.

8. Farowski F, Cornely OA, Vehreschild JJ, Hartmann P, Bauer T, Steinbach A, et al. Quantitation of azoles and echinocandins in compartments of peripheral blood by liquid chromatographytandem mass spectrometry. Antimicrob Agents Chemother 2010;54:1815-9.

9. Decosterd LA, Rochat B, Pesse B, Mercier T, Tissot F, Widmer N, et al. Multiplex ultra-performance liquid chromatography-tandem mass spectrometry method for simultaneous quantification in human plasma of fluconazole, itraconazole, hydroxyitraconazole, posaconazole, voriconazole, voriconazole-N-oxide, anidulafungin, and caspofungin. Antimicrob Agents Chemother 2010;54:5303-15.

10. Egle H, Trittler R, Kümmerer K. An advanced double columnswitching technique (LC-LC) for liquid chromatography/electrospray ionisation tandem mass spectrometry for fully automated 
analysis of caspofungin. Rapid Commun Mass Spectrom 2004;18:2871-7.

11. Neoh CF, He H, Li J, Fullinfaw RO, Leung L, Misra A, et al. Rapid and sensitive liquid chromatography/mass spectrometry assay for caspofungin in human aqueous humor. Antimicrob Agents Chemother 2010;54:4467-70.

12. Schwartz M, Kline W, Matuszewski B. Determination of a cyclic hexapeptide (L-743 872), a novel pneumocandin antifungal agent in human plasma and urine by high-performance liquid chromatography with fluorescence detection. Anal Chim Acta 1997;352:299-307.

13. Traunmüller F, Steiner I, Zeitlinger M, Joukhadar C. Development of a high-performance liquid chromatography method for the determination of caspofungin with amperometric detection and its application to in vitro microdialysis experiments. J Chrom B 2006;843:142-6. 